\title{
Effect of Surface Charge Density on the Affinity of Oxide Nanoparticles for the Vapor-Water Interface
}

\author{
Matthew A. Brown, ${ }^{* \dagger}{ }^{\dagger}$ Nicolas Duyckaerts, ${ }^{\dagger}$ Amaia Beloqui Redondo ${ }^{\dagger}$ Inga Jordan, ${ }^{\ddagger}$ Frithjof Nolting, \\ Armin Kleibert, ${ }^{\S}$ Markus Ammann, ${ }^{\S}$ Hans Jakob Wörner, Jeroen A. van Bokhoven, ${ }^{\dagger}$, \\ and Zareen Abbas*,ll \\ ${ }^{\dagger}$ Institute for Chemical and Bioengineering, ETH Zürich, CH-8093 Zürich, Switzerland \\ ${ }^{\ddagger}$ Laboratory of Physical Chemistry, ETH Zürich, CH-8093 Zürich, Switzerland \\ ${ }^{\S}$ Paul Scherrer Institute, CH-5232 Villigen PSI, Switzerland \\ "Department of Chemistry and Molecular Biology, University of Gothenburg, SE-41296 Gothenburg, Sweden
}

Supporting Information

ABSTRACT: Using in-situ X-ray photoelectron spectroscopy at the vapor-water interface, the affinity of nanometer-sized silica colloids to adsorb at the interface is shown to depend on colloid surface charge density. In aqueous suspensions at $\mathrm{pH} 10$ corrected Debye-Hückel theory for surface complexation calculations predict that smaller silica colloids have increased negative surface charge density that originates from enhanced screening of deprotonated silanol groups $\left(\equiv \mathrm{Si}-\mathrm{O}^{-}\right)$by counterions in the condensed ion layer. The increased negative surface charge density results in an electrostatic repulsion from the vapor-water interface that is seen to a lesser extent for larger particles that have a reduced charge density in the XPS measurements. We compare the results and interpretation of the in-situ XPS and corrected Debye-Hückel theory for surface complexation calculations with traditional surface tension

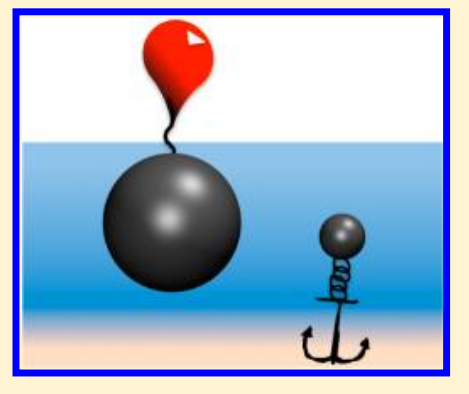
measurements. Our results show that controlling the surface charge density of colloid particles can regulate their adsorption to the interface between two dielectrics.

\section{INTRODUCTION}

The vapor-water-colloid three-way interface is one of extreme complexity that makes developing accurate theoretical models of particle attachment difficult. At the same time, experiments that can probe the three-way interface with molecular level detail are limited and therefore provide infrequent confirmation of theoretical predictions and the validity of the assumptions that go into particle attachment models. The need to improve our molecular level understanding of colloid particle attachment is illustrated by the recent observation that the diffusion of charged nanoparticles at the vapor-water interface is not invariant under a charge reversal of the particles, ${ }^{1}$ which appears at odds with one of the fundamental laws of physics: the invariance of classical phenomena under charge reversal.

Recently, May and co-workers ${ }^{2}$ used a classical nonlinear continuum Poisson-Boltzmann model that assumed (i) the vapor-water interface carries a constant negative potential, (ii) the vapor-water interface remains perfectly flat, (iii) the electrostatic field outside the aqueous region is negligibly small, and (iv) that hysteresis of the contact angle on the particle surface is negligible to, in part, predict that colloid particle adsorption to the vapor-water interface depends strongly on the particles surface charge density (SCD). Their model showed that particles with a higher negative SCD are electrostatically repelled more strongly from the interface, whereas particles with a lower negative SCD can adsorb closer.
Experimentally, the adsorption of colloid particles to the vapor-water interface is most often determined by surface tension measurements and reported as a surface pressure. The surface pressure is given by the difference in the surface tension of the clean (no particle) interface to that of the solution in the presence of the colloid. ${ }^{3}$ Interpreting the meaning of surface pressure in terms of particle adsorption to the interface, either qualitatively or quantitatively, is significantly more challenging than performing the experiment itself and remains debated in the literature. ${ }^{4}$ The general approach is rather simple and goes as follows: the higher (more positive) the surface pressure, the more pronounced the adsorption of the colloid particle to the interface. ${ }^{5,6}$ While not quantitative, this approach has proven relatively reliable and has been used to generate a macroscopic description of particle attachment to the vapor-water interface. The effect of SCD on the affinity of oxide nanoparticles for the vapor-water interface has not been previously reported using surface tension measurements, and therefore the model of May and co-workers ${ }^{2}$ remains to be validated.

Herein, we experimentally determine the adsorption of sizedependent (negative) charge-stabilized colloidal silica nanoparticles to the vapor-water interface using in-situ X-ray photoelectron spectroscopy (XPS) from a liquid microjet. ${ }^{7-9}$

Received: February 6, 2013

Revised: March 17, 2013

Published: March 27, 2013 
The liquid microjet has been in use for nearly 20 years ${ }^{10}$ and is well established for in-situ photoelectron spectroscopy measurements of solutes and solvents at the vapor-water interface. By contrast, the use of the liquid microjet in colloidal science is a relatively young discipline that is still establishing itself as a powerful experimental tool that can provide molecular level insight into geometric, spatial, and electronic structures of solvated colloidal particles. ${ }^{1-13}$ Our in-situ XPS measurements reveal a preferential adsorption of larger silica nanoparticles to the vapor-water interface compared to the smaller ones. The experimental results are complemented by corrected Debye-Hückel theory for surface complexation ${ }^{14,15}$ calculations that reveal an increased SCD for the smaller silica nanoparticles in aqueous suspensions at the $\mathrm{pH}$ of the experiment. We subsequently compare the results and interpretation of the in-situ XPS and corrected Debye-Hückel theory for surface complexation calculations with traditional surface tension measurements using a Wilhelmy plate tensiometer. Our results provide experimental evidence of the preferential exclusion from the vapor-water interface of colloid particles with a higher negative SCD.

\section{EXPERIMENTAL SECTION}

Transmission Electron Microscopy (TEM). The sizes of two commercially available monodisperse silica NP suspensions (Ludox SM-30 and Ludox TM-50) were measured with a FEI Tecnai F30 FEG transmission electron microscope $(300 \mathrm{kV})$ by drying a drop of a $3 \mathrm{wt}$ $\%$ suspension on a copper TEM grid with carbon film support. The suspension $\mathrm{pH}$ was first adjusted to 10 before drying. The images were evaluated by Image J software. Particle size distributions were calculated using 116 SM-30 particles and 312 TM-50 particles. Representative micrographs and the particle size distributions are shown in Figure 1. The particles mean diameters were determined to

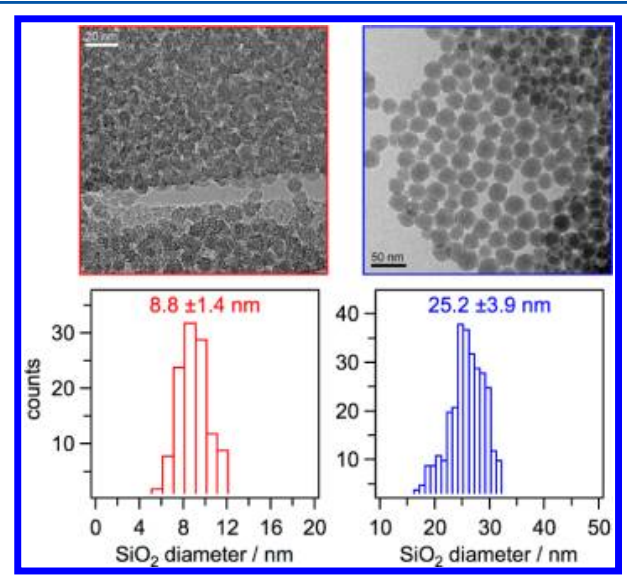

Figure 1. Silica particle size distributions measured by TEM. The mean value for each nanoparticles suspension is shown.

be $8.8 \pm 1.4$ and $25.2 \pm 3.9 \mathrm{~nm}$, respectively. For simplicity, the particles will be referred to as having the nearest whole number, 9 and $25 \mathrm{~nm}$ diameters throughout.

X-ray Photoelectron Spectroscopy (XPS). The XPS experiments were performed at the SIM beamline ${ }^{16}$ of the Swiss Light Source (SLS) using a $35 \mu \mathrm{m}$ liquid jet ${ }^{7-9}$ operating at $279 \mathrm{~K}$ and a flow rate of $0.75 \mathrm{~mL} / \mathrm{min}$. A complete description of in situ XPS at the three-way interface of colloidal NPs in liquid solutions is given elsewhere. ${ }^{11}$ The two silica suspensions were prepared at $2.0 \mathrm{wt} \%$ by diluting commercially available Ludox SM-30 and TM-50. Each suspension was adjusted to have a $\mathrm{NaCl}$ electrolyte concentration of $0.05 \mathrm{M}$ and a $\mathrm{pH}$ of $10.0 \pm 0.2$ using $\mathrm{NaOH}$. The valence band (VB) and $\mathrm{Si} 2 \mathrm{p}$ regions were recorded using an incident photon energy of
$495 \mathrm{eV}$, corresponding to a photoelectron kinetic energy (pKE) of 485 $\mathrm{eV}(\mathrm{VB})$ and $387 \mathrm{eV}$ (Si 2p). The limited escape depth of the photoelectrons from condensed matter ensures the surface sensitivity of the experiment and limits the probe depth to several nanometers. ${ }^{17}$ The total resolution of the beamline and analyzer was $0.25 \mathrm{eV}$.

Surface Tension (ST). Surface tension measurements were performed using an Attension Sigma 702 tensiometer and a platinum Wilhelmy plate. The Wilhelmy plate was cleaned by first rinsing it in Milli- $Q$ water and then in ultrapure ethanol before being heated in a butane flame for $10 \mathrm{~s}$. The balance of the tensiometer was calibrated every morning prior to the measurements. The two Ludox silica suspensions (SM-30 and TM-50) were diluted to the desired wt \% in glass flasks using Milli-Q water prepared fresh everyday. Surface tension measurements of the silica suspensions were performed by averaging 48 dip cycles of the Wilhelmy plate. Measurements were repeated in triplicate to ensure accuracy. The same measurement procedure was followed for solutions of pure water that were always recorded immediately prior to that of the silica suspension. The reported surface pressure as a function of weight percent silica is calculated as follows: $\Pi=\gamma_{\text {water }}-\gamma_{\text {silica }}$. The error bars represent the standard deviation of the measurements.

\section{THEORETICAL SECTION}

Corrected Debye-Hückel theory of surface complexation (CDH-SC) is well suited for predicting particle size and $\mathrm{pH}$ dependent surface charging of nanoparticles in solution. Here we provide a brief description of the relevant features of $\mathrm{CDH}$ SC theory. A complete description can be found elsewhere. ${ }^{14,15}$ In $\mathrm{CDH}$-SC theory a metal oxide nanoparticle is modeled as a sphere having randomly distributed surface hydroxyl sites $(-\mathrm{OH})$ of only one type. Charging of the nanoparticle is described by protonation and deprotonation events of these surface hydroxyl groups. A three-dimensional harmonic oscillator models the binding of the proton to the hydroxyl group where the interacting proton has a specific frequency $(h \nu)$ and interaction energy $\left(\mu_{0}\right)$. Here $h$ is Planck's constant. To obtain the correct $\mathrm{pH}_{\mathrm{PZC}}(\mathrm{pH}$ at the point of zero charge) of a specific metal oxide, the interaction energy $\left(\mu_{0}\right)$ is varied while the frequency $(\nu)$ is kept constant.

A layer of water that has the bulk dielectric constant of water, 78.40 at $25^{\circ} \mathrm{C}$, hydrates the charged NP surface. The screening of the charged NP surface in an electrolyte solution is described by the formation of a diffuse double layer. However, as the surface charge increases a condensed counterion $\left(\mathrm{I}_{\mathrm{C}}\right)$ layer starts to form within the diffuse double layer. The criterion for the formation of the $\mathrm{I}_{\mathrm{C}}$ layer is that when the potential energy of the charged surface exceeds $k_{\mathrm{B}} T$, the $\mathrm{I}_{\mathrm{C}}$ layer starts to form. In the $\mathrm{I}_{\mathrm{C}}$ layer ion correlation effects are accounted for via an excluded volume due to the finite size of the counterions as well as by a corresponding hole correction to the electrostatic repulsion energy. A consequence of the formation of $\mathrm{I}_{\mathrm{C}}$ layer is that the apparent bare surface charge is reduced due to the screening by counterions. The interaction of this effective charged surface with the bulk electrolyte is then obtained by linear response approximation in the corrected Debye-Hückel theory, which accounts for the size of the screening ions. The $\mathrm{CDH}-\mathrm{SC}$ theory is formulated in terms of a density functional for the free energy. The properties of charged particles immersed in an electrolyte solution such as the surface charge density, effective charge, and thickness of the $\mathrm{I}_{\mathrm{C}}$ layer are obtained by minimizing the free energy density functional.

\section{RESULTS AND DISCUSSION}

Figures $2 \mathrm{a}$ and $2 \mathrm{~b}$ show the $\mathrm{Si} 2 \mathrm{p} \mathrm{XP}$ spectra recorded at the vapor-water interface for silica suspensions of 9 and $25 \mathrm{~nm}$ 


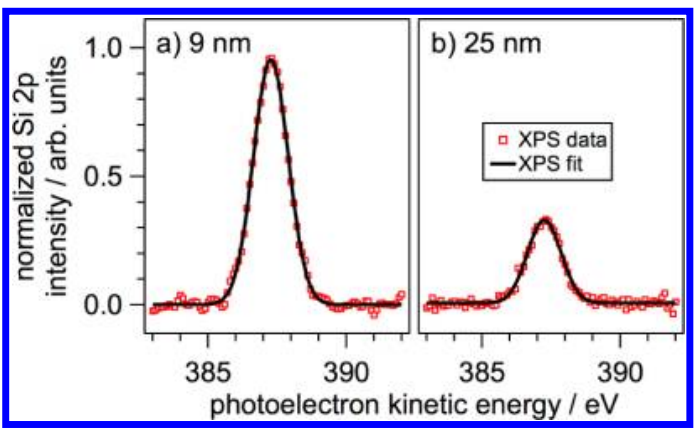

Figure 2. $(\mathrm{a}, \mathrm{b})$ Normalized Si $2 \mathrm{p}$ XP spectra for $2.0 \mathrm{wt} \%$ suspensions of 9 and $25 \mathrm{~nm}$ silica particles, respectively. The spectra were collected using the liquid microjet with incident photon energy of $495 \mathrm{eV}$. Both spectra have been fitted using a single Gaussian function (solid black line) with a full width at half-maximum of $1.4 \mathrm{eV}$. The normalized signal intensity from the $25 \mathrm{~nm}$ suspension is $32 \%$ that of the $9 \mathrm{~nm}$ suspension.

particles, respectively (red open symbols). The spectra were recorded using the liquid microjet $^{7-9}$ at a fixed solution concentration of 2.0 wt \% silica and $0.05 \mathrm{M} \mathrm{NaCl}$ and at $\mathrm{pH}$ $10.0 \pm 0.2$. The XP spectra are each well fitted using a single Gaussian having a full width at half-maximum (fwhm) of $1.4 \mathrm{eV}$ (solid black line). The integrated areas of the Si $2 p$ spectra have been normalized to the integrated area of the $1 b_{1}$ orbital of water (the solvent, not shown), which is recorded immediately prior to and after the collection of the $\mathrm{Si} 2 \mathrm{p}$ region. Normalizing in this fashion ensures that any variation in the spatial overlap of the synchrotron radiation with the liquid microjet that may occur over time does not contribute to our findings. The $\mathrm{Si} 2 \mathrm{p}$ spectra are presented on a relative scale where the maximum intensity of the $9 \mathrm{~nm}$ silica particles has been set to unity. The recorded $\mathrm{Si} 2 \mathrm{p}$ XP intensity from the suspension of $25 \mathrm{~nm}$ particles is $32 \%$ of that from the $9 \mathrm{~nm}$ particles suspension.

The decreased signal intensity from the suspension of the 25 $\mathrm{nm}$ particles, which have a reduced surface-to-volume ratio compared with the $9 \mathrm{~nm}$ particles, is not unexpected in an XPS experiment. To quantify the drop in the signal intensity brought about by the decrease in the surface-to-volume ratio, we have calculated the predicted Si $2 p$ XPS signal intensities when both the 9 and $25 \mathrm{~nm}$ particles have the same physical distribution in suspension; that is, the top surfaces of both particles are at the same depth below the flat vapor-water interface (see Figure 3). The calculation is valid for virtually any distance into suspension, including when the top surface of the particles are in contact (shown) with the vapor-water interface (a depth corresponding to $z=0$ ). The predicted XPS signal intensities are calculated ${ }^{18}$ using $\int \mathrm{e}^{-z / \mathrm{IMFP}} \rho(z) \mathrm{d} z$ and normalized to the number of particles in suspension (there are $21.4 \times$ more $9 \mathrm{~nm}$ particles) assuming a spherical shape, where $z$ is the distance into suspension below the vapor-water interface, IMFP is the inelastic mean free path of the experiment, and $\rho(z)$ is the silica NP density at a given depth into the suspension. The XPS experiment was performed at a photoelectron kinetic energy of $387 \mathrm{eV}$, which corresponds to an IMFP of $1.7 \mathrm{~nm}$ in silica. ${ }^{17}$ The calculation assumes a constant photoelectron attenuation by the solvent above the NP that is equal to the IMFP in silica. However, because the escape depth of photoelectrons through liquid water is not well understood, we have also calculated the predicted XP signal intensities for IMFP's of $\pm 30 \%$. For particle suspensions with uniform physical distributions at the vaporwater interface our calculations predict the signal intensity of the $25 \mathrm{~nm}$ particles to be $17 \%$ of that for the suspension of 9 $\mathrm{nm}$ particles. Changing the IMFP of the experiment by $\pm 30 \%$ has very little effect $(-1 \%$ for an IMFP of $-30 \%$ and $+3 \%$ for an IMFP of $+30 \%$ ) on the results, and we can therefore disregard the uncertainty of photoelectron attenuation by water influencing our results in this case.

It is immediately clear that the experimentally recorded intensity from the suspension of $25 \mathrm{~nm}$ particles (32\%) is ca. $2 \times$ higher than that predicted $(17 \%)$ by the model. To reproduce the experimental intensities, a different physical distribution of the two sized NPs at the vapor-water interface must exist. A physical distribution that can account for the recorded XPS intensities at the vapor-water interface requires that the $9 \mathrm{~nm}$ particles reside on average $1.0 \mathrm{~nm}$ below that of the $25 \mathrm{~nm}$ particles (Figure 4), effectively increasing the relative signal intensity of the larger $25 \mathrm{~nm}$ particles. Our experiment cannot determine the absolute distance that the NPs reside below the vapor-water interface because of uncertainties in the

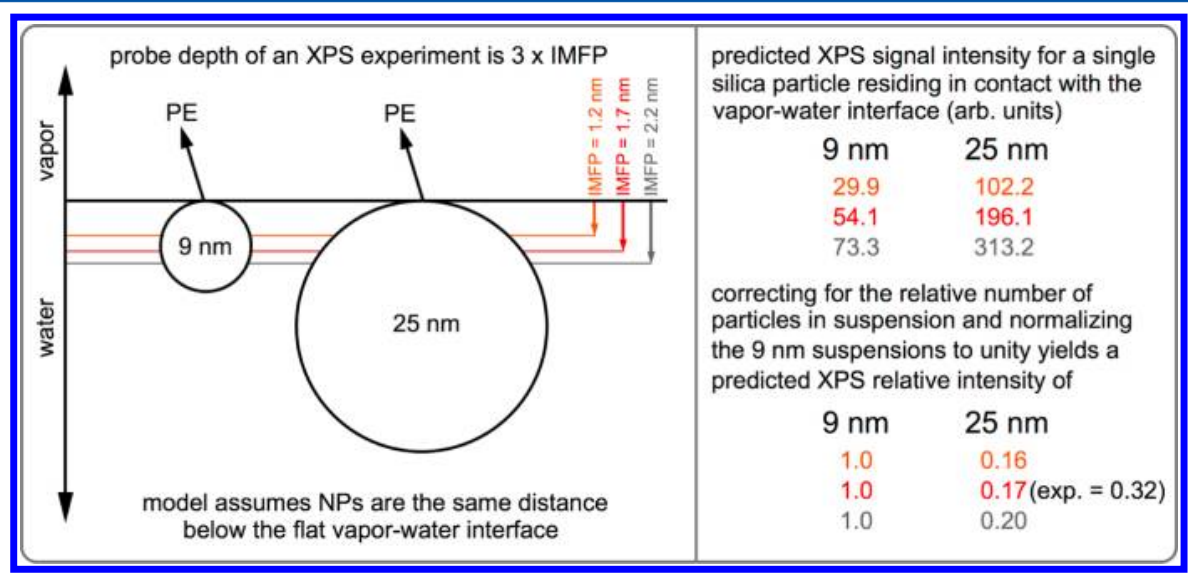

Figure 3. A model that assumes the 9 and $25 \mathrm{~nm}$ silica particles have identical physical distributions at the vapor-water interface cannot reproduce the experimental XP signal intensities shown in Figure 2. Here we have calculated the predicted XP intensities using the physical distribution shown on the left. In this geometry, and normalized to the number of particles in suspension the $25 \mathrm{~nm}$ sample would be expected to yield $17 \%$ the signal intensity of the $9 \mathrm{~nm}$ particle suspension. By contrast, the experimental intensity is $32 \%$. The calculation is valid for virtually any distance the NPs are placed below the (flat) vapor-water interface provided the tops of the particles are at an identical depth. There is very little influence on the predicted signal intensity if the IMFP is allowed to vary by $\pm 30 \%$. 


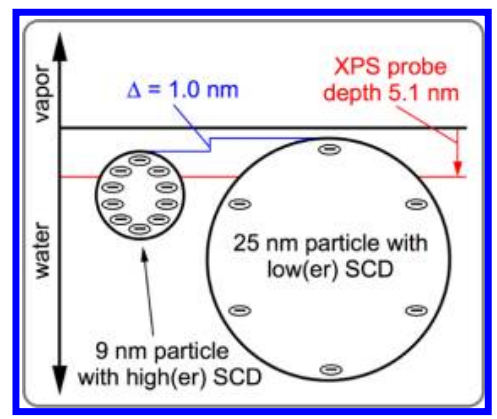

Figure 4. A model of particle attachment to the (flat) vapor-water interface that can reproduce the experimental XP intensities requires that the $9 \mathrm{~nm}$ particles reside on average $1.0 \mathrm{~nm}$ below the larger 25 $\mathrm{nm}$ particles. The XPS probe depth is $3 \times$ the inelastic mean free path of the experiment $(1.7 \mathrm{~nm})$.

IMFP through liquid water, but it should be noted that the recorded XP signal intensities are consistent only with the NPs being fully solvated (i.e., the top of the NP does not protrude into the vapor phase). This observation is in agreement with the hydrophilic nature of charge-stabilized silica at $\mathrm{pH} 10$ as evidenced by surface tension measurements ${ }^{4,19}$ (vide infra).

Using corrected Debye-Hückel theory for surface complexation, ${ }^{14,15}$ the SCD of 9 and $25 \mathrm{~nm}$ silica particles has been calculated in $0.05 \mathrm{M} \mathrm{NaCl}$ (Figure 5). The model assumes

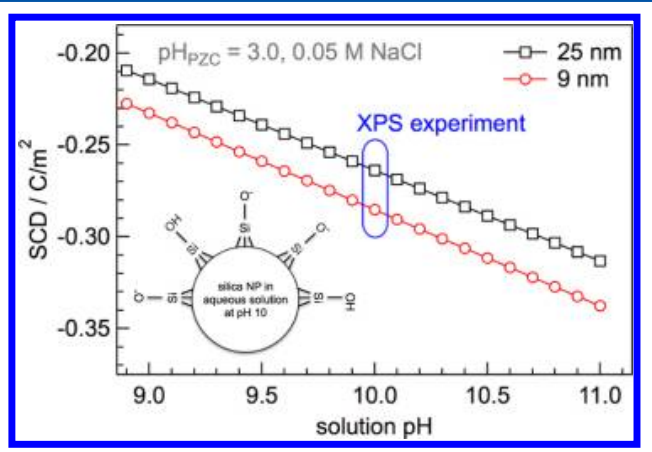

Figure 5. Surface charge density (SCD) of 9 and $25 \mathrm{~nm}$ silica particles in $0.05 \mathrm{M} \mathrm{NaCl}$ as calculated by corrected Debye-Hückel theory for surface complexation.

spherical particles with a random distribution of $6 \mathrm{OH}$ sites/ $\mathrm{nm}^{2}$ at the surface. ${ }^{20,21}$ The point of zero charge $\left(\mathrm{pH}_{\mathrm{PZC}}\right)$ of silica was fixed to $3.0 .^{22}$ The calculation predicts an increased (more negative) SCD for the $9 \mathrm{~nm}$ particles $\left(-0.29 \mathrm{C} / \mathrm{m}^{2}\right)$ compared with the $25 \mathrm{~nm}$ particles $\left(-0.26 \mathrm{C} / \mathrm{m}^{2}\right)$ at the $\mathrm{pH}$ of the experiment that results from the increased radius of curvature at the water $-9 \mathrm{~nm}$ particle interface. As the particles become smaller, increased coadsorption of $\left(\mathrm{Na}^{+}\right)$counterions in the condensed ion layer facilitates the deprotonation of surface hydroxyl groups by providing enhanced screening of the charged sites. ${ }^{14,15,23}$ The increased SCD of the $9 \mathrm{~nm}$ particles can also be rationalized due to a decrease in the attractive van der Waals potential as the radius of curvature of the NP increases the separation between adjacent $\equiv \mathrm{Si}-\mathrm{O}^{-}$charge groups by as much as $6 \%$ compared with larger $(25 \mathrm{~nm})$ particles $^{24}$ and decreases the potential energy by up to $20 \%{ }^{25}$ The negative potential ${ }^{26}$ and absolute scale ${ }^{27}$ of the SCD predicted by our model agree well with those experimentally determined for silica colloids of similar size and structure.

An electrostatic screening effect brought about by the increased radius of curvature of smaller particles has been previously used to explain decreased acidity at the water-metal oxide interface of smaller-compared with larger- $\gamma-\mathrm{Fe}_{2} \mathrm{O}_{3}$ nanoparticles in aqueous suspensions at $\mathrm{pH}$ above the $\mathrm{pH}_{\mathrm{PZC}}$ of maghemite. ${ }^{23}$ Our calculations predict that for a given $\mathrm{pH}$ above the $\mathrm{pH}_{\mathrm{PZC}}$ of silica the acid-base equilibrium

$$
\equiv \mathrm{Si}-\mathrm{OH}+\mathrm{OH}^{-} \Leftrightarrow \equiv \mathrm{Si}-\mathrm{O}^{-}+\mathrm{H}_{2} \mathrm{O}
$$

is shifted more toward the right $\left(\equiv \mathrm{Si}-\mathrm{O}^{-}\right)$for smaller silica particles than for larger particles, effectively decreasing the acidity at the aqueous-silica nanoparticle interface. The increased concentration of deprotonated surface hydroxyl groups on the smaller silica particles results in an increased SCD (in this case the SCD will become more negative). These findings are in agreement with in-situ X-ray absorption spectroscopy experiments that postulated smaller silica colloids undergo a larger fraction of deprotonation $\left(\equiv \mathrm{Si}-\mathrm{O}^{-}\right)$and protonation $\left(\equiv \mathrm{Si}-\mathrm{OH}_{2}^{+}\right.$) events at high and low $\mathrm{pH}$, respectively, than do larger sized particles. ${ }^{26}$

The interaction of surfactant-free negatively charged colloidal particles with the vapor-water interface causes an electrostatic repulsion that preferentially excludes the smaller particles (with a more negative SCD) from the interface toward bulk solvation. In the case of the silica particles studied herein, where the surface of the particle is decorated in a combination of $\equiv \mathrm{Si}-$ $\mathrm{OH}$ and $\equiv \mathrm{Si}-\mathrm{O}^{-}$functionalities at $\mathrm{pH} 10$ (see inset of Figure $5)$, the in-situ XPS measurements at the vapor-water interface reveal that the top of the $9 \mathrm{~nm}$ particles are located on average $1.0 \mathrm{~nm}$ further into solution than that of the $25 \mathrm{~nm}$ particles (Figure 4). However, because the SCD is a strong function of suspension $\mathrm{pH}$ (Figure 5), our results also suggest that the adsorption of colloidal particles to the vapor-water interface can be regulated on a microscopic level by carefully engineering the appropriate surface structure, with a well-defined response to suspension $\mathrm{pH}$, into the particle.

As was mentioned in the Introduction, surface tension measurements are the most common analytical tool used to experimentally determine particle attachment to the vaporwater interface. We have performed surface tension measurements on the two different sized silica colloids used in the insitu XPS measurements. The surface pressure as a function of silica wt \% is shown in Figure 6. In agreement with the hydrophilic nature of charge-stabilized silica NPs at high $\mathrm{pH}$, the surface pressure as a function of NP concentration, while becoming more positive with increasing concentration, is near to zero. That is, the surface tension of these suspensions does

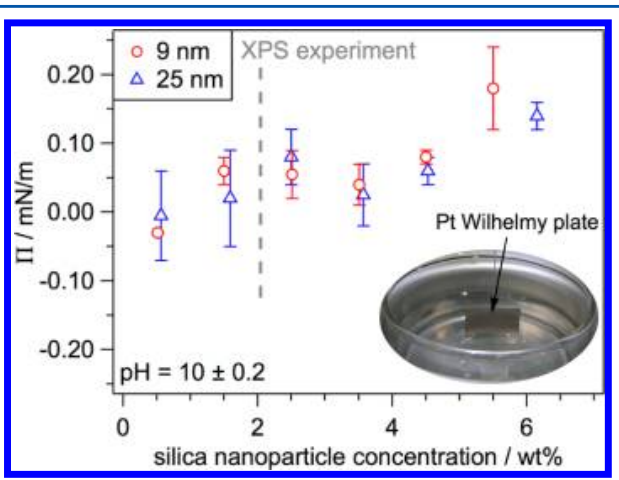

Figure 6. Surface pressure measurements of 9 and $25 \mathrm{~nm}$ silica particle suspensions as a function of concentration. The measurements were performed using a platinum Wilhelmy plate at $\mathrm{pH} 10$. The error bars represent the standard deviations of the measurements. 
not vary to an appreciable extent from that of the neat water and is in agreement with previous surface tension studies of nanometer-sized charge-stabilized colloidal silica at high $\mathrm{pH}$, $^{4,19}$ Over the concentration range $0.5-6$ wt $\%$ the surface pressure of both sized silica NP suspensions are within the reproducibility of the measurements, which would suggest that there is no preferential adsorption of one size of NP over the other to the vapor-water interface. It is clear that at concentrations in and around that used for the in-situ XPS measurements the surface tension measurements do not show a change in surface pressure that would be traditionally interpreted to mean a preferential exclusion of the $9 \mathrm{~nm}$ particles from the vapor-water interface (as seen by XPS).

At high $\mathrm{pH}$ in aqueous solution silica NPs are hydrophilic. The two predominant surface functionalities ${ }^{12,19,28}$ at $\mathrm{pH}$ units far above the $\mathrm{pH}_{\text {PZC }}$ are $\equiv \mathrm{Si}-\mathrm{OH}$ and $\equiv \mathrm{Si}-\mathrm{O}^{-}$which act to drive the NPs away from the interface and into bulk solvation. This hydrophilic character of silica at $\mathrm{pH} 10$ is clearly represented in the surface pressure measurements of Figure 6. Near zero surface pressure $\left(\Pi=\gamma_{\text {water }}-\gamma_{\text {silica }}\right)$ is most easily interpreted in a macroscopic picture as meaning the NPs are excluded from the vapor-water interface. Our surface pressure measurements and those of others ${ }^{4,19}$ fully support this description. The microscopic picture of particle attachment to the vapor-water interface provided by our in-situ XPS measurements does, however, not support the macroscopic description provided by surface tension measurements. The XPS experiment is inherently surface sensitive with IMFPs on the order of several nanometers and would therefore imply that some silica NPs are in the vicinity of the vapor-water interface (because we observe a Si $2 p$ XPS signal). If we assume the probe depth of the experiment to be represented by 3 times the IMFP, a finite concentration of hydrophilic silica NPs are contained roughly within the outermost ten water layers at the vapor-water interface.

If our argument and reasoning are valid, then not only does a finite amount of silica NPs reside in the outermost layers of suspension but there is also a size-dependent concentration near the vapor-water interface, and the interpretation of the surface pressure and XPS experiments appear to be at odds with one another. To resolve this ambiguity, we propose a revised interpretation of zero surface pressure, meaning the exclusion of particles from the vapor-water interface. Instead of directly measuring particle adsorption, surface pressure measurements are more likely reflecting any changes in the hydrogen-bonding network of water at the vapor-water interface brought about by particle adsorption. The similarity between the surface pressures for both sized NPs would suggest that the hydrogen-bonding network of water at the vaporwater interface is equally affected (in this case unaffected) and independent of particle size and SCD. This statement warrants a certain level of clarification given the hydrophilic nature of silica at $\mathrm{pH} 10$ and the high radius of curvature of the particles used in this study. Water is known to hydrogen bond in two different configurations with surface silanol groups. ${ }^{29}$ With the acidic out-of-plane silanol water acts as a hydrogen bond acceptor, whereas with the in-plane silanol water acts as the hydrogen bond donor. We would expect that the increased SCD and radius of curvature as well as increased coadsorption of counterions (each of which will be solvated in their own right) in the condensed ion layer of smaller silica particles would result in a different hydrogen bonding structure of water at the surface of the particles. This change in the hydrogen- bonding network of water, if it was pronounced, and at the vapor-water interface should then manifest itself as a change in the surface pressure. The fact that it does not clearly reflects the physical distribution and radius of curvature of these silica particles in suspension. The hydrophilic nature of these particles almost certainly assures that a few layers of water are between the particles surface and the vapor-water interface. The high radius of curvature of nanometer-sized particles also greatly reduces any effect the particles have on the hydrogenbonding network at the vapor-liquid interface. Even if the larger silica particles were to adsorb with their top surface in contact with the interface (see Figure 3), the radius of curvature prevents the particles from occupying more that a few percent of the outermost few molecular layers. The small concentration of silica in the outermost few molecular layers of suspension does not appear to be enough to cause a change in the surface pressure at the vapor-water interface. At $\mathrm{pH} 10$, the (relatively low) concentrations studied here, and for particles with diameters on the nanoscale, the surface pressure measurements are unable to reproduce the molecular level detail provided by in-situ XPS and fail to demonstrate the preferential exclusion of smaller sized colloids with increased (negative) SCD over larger particles that have an reduced (negative) SCD.

Returning to the model of particle attachment at the vaporwater interface of May and co-workers, ${ }^{2}$ one of their assumptions was that the vapor-water interface carries a negative potential, which was predicted to interact with the negative surface charge of their model particles and result in an electrostatic repulsion that favors bulk solution. The degree of repulsion was shown to increase with an increase in the particles negative SCD. It should be noted that the surface potential of the vapor-water interface is a widely debated topic in the literature, ${ }^{30,31}$ and for each report that asserts negative potential $^{32}$ another can be found that argues the opposite. ${ }^{33}$ Studies such as ours may be able to shed some light on this controversial subject. At $\mathrm{pH} 10$ and in low concentration (0.05 M) monovalent $\left(\mathrm{Na}^{+}, \mathrm{Cl}^{-}\right)$electrolyte solution silica colloids do not undergo charge inversion, ${ }^{34,35}$ in agreement with previously reported zeta potential measurements ${ }^{26}$ and our corrected Debye-Hückel theory for surface complexation ${ }^{14,15}$ calculations. In addition, monovalent $\mathrm{Na}^{+}$counterions do not completely screen the surface charge as evidenced by the particles stability. Complete screening of the surface charge would result in decreased interparticle repulsion and cause the silica suspension to gel. Under the conditions of our experiment, the potential experienced several angstroms from the surface of the particles is negative and would therefore suggest based on the interpretation of the in-situ XPS measurements that the vapor-water interface of dilute 0.05 $\mathrm{M} \mathrm{NaCl}$ does indeed have a negative surface potential. ${ }^{36}$

\section{CONCLUSIONS}

The SCD of oxide particles in aqueous suspensions has been predicted using corrected Debye-Hückel theory for surface complexation to be a function of both physical diameters, increasing as particle diameters decrease, and of suspension $\mathrm{pH}$, increasing as $\mathrm{pH}$ is shifted away from the $\mathrm{pH}_{\mathrm{PZC}}$. In-situ XPS measurements at the vapor-water interface have shown that the increased negative SCD of smaller silica colloids at high $\mathrm{pH}$ results in a preferential exclusion of the particle from the vapor-water interface. The origin of this repulsion is electrostatic and points toward the vapor-water interface having a negative potential. Silica particles with diameters of 25 
$\mathrm{nm}$ have reduced negative SCD and were shown to adsorb in closer proximity to the vapor-water interface.

Our in-situ XPS measurements using the liquid microjet also highlight the benefits quantitative physical probes can provide for generating a microscopic picture of particle attachment to the vapor-water interface. Traditional surface tension measurements were also performed and are unable to provide the same molecular level detail in-situ XPS affords.

\section{ASSOCIATED CONTENT}

\section{(S Supporting Information}

Surface charge density curves as a function of solution $\mathrm{pH}(0-$ 11) and electrolyte concentration $(0.01$ and $0.05 \mathrm{M} \mathrm{NaCl})$ for silica particle sizes between 1 and $100 \mathrm{~nm}$. This material is available free of charge via the Internet at http://pubs.acs.org.

\section{AUTHOR INFORMATION}

\section{Corresponding Author}

*E-mail: matthew.brown@chem.ethz.ch (M.A.B.); zareen@ chem.gu.se (Z.A.).

\section{Notes}

The authors declare no competing financial interest.

\section{ACKNOWLEDGMENTS}

Part of this work was performed at the Swiss Light Source, Paul Scherrer Institute, Villigen, Switzerland. Financial support of the NAPP endstation is provided through PSI FoKo and SNF R'Equip (grant no. 139139). Z.A. acknowledges financial support from Kungl. Vetenskaps och Vitterhets-Samhället i Göteborg and from Formas through the Nanosphere centre of excellence at the University of Gothenburg. We are grateful to Dr. Krumeich for the TEM images.

\section{REFERENCES}

(1) Gehring, T.; Fischer, T. M. Diffusion of nanoparticles at an air/ water interface is not invariant under a reversal of the particle charge. $I$. Phvs. Chem. C 2011, 115, 23677-23681.

(2) Shrestha, A.; Bohinc, K.; May, S. Immersion depth of positively versus negatively charged nanoparticles at the air-water interface: $\mathrm{A}$ Poisson-Boltzmann model. Langmuir 2012, 28, 14301-14307.

(3) Barnes, G. T. Interfacial Science: An Introduction, 2nd ed.; Oxford University Press: New York, 2011.

(4) Blute, I.; Pugh, R. J.; van de Pas, J.; Callaghan, I. Industrial manufactured silica nanoparticle sols. 2: Surface tension, particle concentration, foam generation and stability. Colloids Surf. A 2009, $337,127-135$.

(5) Dong, L. C.; Johnson, D. T. The study of the surface tension of charge-stabilized colloidal dispersions. I. Dispersion Sci. Technol. 2004, $25,575-583$.

(6) Okubo, T. Surface-tension of structured colloidal suspensions of polystyrene and silica spheres at the air-water interface. $\underline{\text { L. Colloid }}$ Interface Sci. 1995, 171, 55-62.

(7) Brown, M. A.; D’Auria, R.; Kuo, I.-F. W.; Krisch, M. J.; Starr, D. E.; Bluhm, H.; Tobias, D. J.; Hemminger, J. C. Ion spatial distributions at the liquid-vapor interface of aqueous potassium fluoride solutions. Phvs. Chem. Chem. Phvs. 2008, 10, 4778-4784.

(8) Brown, M. A.; Vila, F.; Sterrer, M.; Thürmer, S.; Winter, B.; Ammann, M.; Rehr, J. J.; van Bokhoven, J. A. Electronic structure of formic acid $(\mathrm{HCOOH})$ and formate $\left(\mathrm{HCOO}^{-}\right)$in aqueous solutions. L. Phys. Chem. Lett. 2012, 3, 1754-1759.

(9) Brown, M. A.; Faubel, M.; Winter, B. X-ray photo- and resonant Auger-electron spectroscopy studies of liquid water and aqueous solutions. Annu. Rep. Prog. Chem. Sect. C 2009, 105, 174-212.
(10) Faubel, M.; Steiner, B.; Toennies, J. P. Photoelectron spectroscopy of liquid water, some alcohols, and pure nonane in free micro jets. I. Chem. Phvs. 1997, 106, 9013-9031.

(11) Brown, M. A.; Jordan, I.; Beloqui Redondo, A.; Kleibert, A.; Wörner, H. J.; van Bokhoven, J. A. In situ photoelectron spectroscopy at the liquid/nanoparticle interface. Surf. Sci. 2013, 610, 1-6.

(12) Brown, M. A.; Seidel, R.; Thürmer, S.; Faubel, M.; Hemminger, J. C.; van Bokhoven, J. A.; Winter, B.; Sterrer, M. Electronic structure of sub-10 nm colloidal silica nanoparticles measured by in situ photoelectron spectroscopy at the aqueous-solid interface. Phvs. Chem. Chem. Phys. 2011, 13, 12720-12723.

(13) Söderstrom, J.; Ottosson, N.; Pokapanich, W.; Öhrwall, G.; Björneholm, O. Functionalized nanoparticles in aqueous surroundings probed by X-ray photoelectron spectroscopy. I. Electron Spectrosc. Relat. Phenom. 2011, 184, 375-378.

(14) Abbas, Z.; Labbez, C.; Nordholm, S.; Ahlberg, E. Sizedependent surface charging of nanoparticles. I. Phys. Chem.C 2008, $112,5715-5723$.

(15) Gunnarsson, M.; Abbas, Z.; Ahlberg, E.; Nordholm, S. Corrected Debye-Huckel analysis of surface complexation III. Spherical particle charging including ion condensation. I. Colloid Interface Sci. 2004, 274, 563-578.

(16) Flechsig, U.; Nolting, F.; Fraile Rodriguez, A.; Krempasky, J.; Quitmann, C.; Schmidt, T.; Spielmann, S.; Zimoch, D. Performance measurements at the SLS SIM beamline. AIP Conf. Proc. 2010, 1234, 319-322.

(17) Powell, C. J.; Jablonski, A. NIST Electron Inelastic-Mean-FreePath Database, Version 1.1; National Institute of Standards and Technology: Gaithersburg, MD, 2000.

(18) Brown, M. A.; Winter, B.; Faubel, M.; Hemminger, J. C. Spatial distribution of nitrate and nitrite anions at the liquid/vapor interface of aqueous solutions. I. Am. Chem. Soc. 2009, 131, 8354-8355.

(19) Ravera, F.; Santini, E.; Loglio, G.; Ferrari, M.; Liggieri, L. Effect of nanoparticles on the interfacial properties of liquid/liquid and

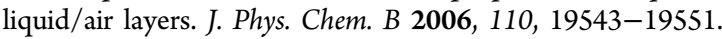

(20) Surface site densities for silica vary in the literature from 5-10 $\mathrm{OH}$ sites $/ \mathrm{nm}^{2}$ depending on the type of silica. Carroll, S. A.; Maxwell, R. S.; Bourcier, W.; Martin, S.; Hulsey, S. Evaluation of silica-water surface chemistry using NMR spectroscopy. Geochim. Cosmochim. Acta 2002, 66, 913-926.

(21) Surface site densities for silica vary in the literature from 5-10 $\mathrm{OH}$ sites $/ \mathrm{nm}^{2}$ depending on the type of silica. Campen, R. K.; Pymer, A. K.; Nihonyanagi, S.; Borguet, E. Linking surface potential and deprotonation in nanoporous silica: Second harmonic generation and acid/base titration. J. Phys. Chem. C 2010, 114, 18465-18473.

(22) Tadros, T. F.; Lyklema, J. Adsorption of potential-determining ions at silica-aqueous electrolyte interface and role of some cations. $\underline{L}$. Electroanal. Chem. 1968, 17, 267-275.

(23) Vayssieres, L. On the effect of nanoparticle size on water-oxide interfacial chemistry. I. Phvs. Chem. C 2009, 113, 4733-4736.

(24) Kamiya, H.; Mitsui, M.; Takano, H.; Miyazawa, S. Influence of particle diameter on surface silanol structure, hydration forces, and aggregation behavior of alkoxide-derived silica particles. J. Am. Ceram. Soc. 2000, 2, 287-293.

(25) Garrone, E.; Kazansky, V. B.; Kustov, L. M.; Sauer, J.; Senchenya, I. N.; Ugliengo, P. Spectroscopic and ab initio study of the interaction of molecular-hydrogen with the isolated silica hydroxyls and related systems. I. Phvs. Chem. 1992, 96, 1040-1045.

(26) Brown, M. A.; Huthwelker, T.; Beloqui Redondo, A.; Janousch, M.; Faubel, M.; Arrell, C. A.; Scarongella, M.; Chergui, M.; van Bokhoven, J. A. Changes in silanol protonation state measured in situ at the silica-aqueous interface. I. Phvs. Chem. Lett. 2012, 3, 231-235.

(27) Bolt, G. H. Determination of the charge density of silica sols. $L$. Phvs. Chem. 1957, 61, 1166-1169.

(28) Zhuravlev, L. T. The surface chemistry of amorphous silica. Zhuravlev model. Colloids Surf. A 2000, 173, 1-38.

(29) Sulpizi, M.; Gaigeot, M.-P.; Sprik, M. The silica/water interface: How the silanols determine the surface acidity and modulate the water properties. I. Chem. Theory Comput. 2012, 8, 1037-1047. 
(30) Zimmermann, R; Freudenberg, U.; Schweiss, R.; Küttner, D.; Werner, C. Hydroxide and hydronium ion adsorption - A survey. Curr. Opin. Colloid Interface Sci. 2010, 15, 196-202.

(31) Kathmann, S. M.; Kuo, I.-F. W.; Mundy, C. J.; Schenter, G. K. Understanding the surface potential of water. I. Phvs. Chem. B 2011, 115, 4369-4377.

(32) Beattie, J. K.; Djerdjev, A. M.; Warr, G. G. The surface of neat water is basic. Faradav Discuss. 2009, 141, 31-39.

(33) Jungwirth, P.; Tobias, D. J. Specific ion effects at the air/water interface. Chem. Rev. 2006, 106, 1259-1281.

(34) Lyklema, J. Overcharging, charge reversal: Chemistry or physics? Colloids Surf. A 2006, 291, 3-12.

(35) Lyklema, J. Quest for ion-ion correlations in electric double layers and overcharging phenomena. Adv. Colloid Interface Sci. 2009, 147-148, 205-213.

(36) Creux, P.; Lachaise, J.; Gracia, A.; Beattie, J. K. Specific cation effects at the hydroxide-charged air/water interface. I. Phys. Chem. C 2007, 111, 3753-3755. 